Instituto Internacional de Investigación y Desarrollo Tecnológico Educativo INDTEC, C.A.

DOI: https://doi.org/10.29394/scientific.issn.2542-2987.2017.2.4.15.265-282

OAI-PMH: http://www.indteca.com/ojs/index.php/Revista Scientific/oai

\title{
Educación en Valores desde el Contexto Universitario
}

Autora: María Lastenia Palacios Palacios Universidad Fermín Toro, UFT marialasteniapalacios@hotmail.com

Lara, Venezuela

\section{Resumen}

La Educación en Valores en el contexto universitario desde la visión del pensamiento complejo, luego de comprender, los significados y sentidos que poseen los docentes con respecto a la educación en valores en su praxis pedagógica. El estudio se desarrolló a través de la metodología de naturaleza cualitativa, fundamentado en el paradigma interpretativo, el cual focaliza su atención en la interpretación de las experiencias y vivencias de los actores sociales. El método empleado fue el método fenomenológico, el cual según Martínez (2004) es donde se articula la hermenéutica al contexto de la temporalidad y la historicidad de la existencia humana. El proceso de recogida de información se llevó a cabo mediante la entrevista en profundidad a cinco (5) actores sociales, quienes se seleccionaron atendiendo al criterio de docentes que se desempeñan en la Universidad estudiada. Para la discusión de los resultados se utilizó la categorización, la triangulación de la información y el proceso de teorización, lo que me permitió construir la teoría emergente, la cual se denominó Construcción Epistemológica de los Valores: Saberes y Haceres.

Palabras clave: educación; valores; universidad. 


\title{
Education in Values from the Context University
}

\begin{abstract}
Values Education in the university context from the perspective of complex thinking, then to understand the meanings and senses that teachers have regarding values education in their pedagogical praxis. The study was conducted through qualitative methodology, based on the interpretive paradigm, which focuses its attention on the interpretation of experiences and experiences of social actors. The method used was the phenomenological method, which according to Martinez (2004) is where hermeneutics is integrated in the context of temporality and historicity of human existence. The information collection process was conducted through in-depth interviews to five (5) social actors, who were selected by the criterion of teachers working at the University studied. categorization, triangulation of information and theorizing process was used for the discussion of the results, which allowed me to build the emerging theory, which was called Construction Epistemology of values: knowledge and practice.
\end{abstract}

Keywords: education; values; university. 


\section{Introducción}

La educación como la vía más valiosa para elevar el nivel cultural de las naciones en el contexto mundial, adquiere gran relevancia en el momento histórico que atraviesa el género humano, signado por impresionantes y vertiginosas transformaciones, que ocurren simultáneamente y que afectan la calidad de vida de las personas. En este sentido, para enfrentar estos escenarios complejos y transformarlos en cotidianidades, precisa generar nuevas teorías, nuevas estrategias y nuevos modos de hacer, para tener una compresión clara de los ambientes interaccionantes, que emergen en el mundo globalizado de hoy.

En esta investigación se aborda la problemática de trabajar por una formación no sólo en el dominio de conocimientos científicos, sino también en el dominio del proceso de generación de nuevos conocimientos, en el desarrollo de habilidades y en la formación de valores. Se analizan las características fundamentales del medio social con la que los profesionales van a enfrentarse y las principales metas que deben ser trazadas por el profesor para formar un profesional íntegro con elevada educación y desarrollo de la personalidad que sea capaz de las respuestas a las necesidades de nuestro entorno.

Al profundizar en el análisis de la situación anterior, es indispensable llegar al convencimiento de la necesidad de educar en valores, proceso que se inicia desde los primeros años de vida, correspondiendo en primer lugar a la familia, posteriormente a la escuela y por ende a las instituciones universitarias como entes socializadores por excelencia.

Desde esta perspectiva, el sistema educativo está llamado a repensar sus políticas educativas, asumiendo como reto el fin primordial de la educación que es la formación integral del educando y el fomento de un ciudadano capaz de participar en forma activa y solidaria en el proceso de transformación de la sociedad. Para ello, es necesario asumir que el pleno desarrollo de la 
personalidad, no se logra si no se hace énfasis en una educación basada en valores, que permita avivar además del conocimiento, las actitudes y los valores éticos-morales de los estudiantes de manera que pueda insertarse con éxito en una sociedad cada vez más compleja.

En este sentido, el sistema de educación superior, es un ente constituido para responder a las demandas en todos los órdenes del país; lo que significa, que es una institución que garantiza el progreso y desarrollo del mismo. En correspondencia, debe constantemente ser reorganizado para impulsarlo hacia las transformaciones imprescindibles que la sociedad reclama, para actuar sobre esas necesidades de manera eficaz, eficiente y de calidad.

Estas consideraciones, suponen que la educación superior debe ejecutar una gerencia pertinente con los cambios y exigencias del momento, donde a través de sus funciones básicas: docencia, investigación y extensión dirija sus acciones hacia el logro de formar ciudadanos integrales, donde estos adquieran herramientas fundamentales orientadas a la capacidad de aprender, crear, innovar y comunicar a través de una actitud crítica, reflexiva, analítica, proactiva, democrática y con un sentido de pertinencia social enmarcada dentro de los valores ciudadanos. Sólo de esta manera, la educación superior respondería a los fines por las que fueron creadas, que no es más, la de contribuir con el desarrollo sostenible y sustentable del país.

Al respecto Romero (2004) afirma:

"El desarrollo del país requiere un sistema de educación superior con mayor responsabilidad y mejor calidad, en el que se asegure que los individuos cuenten con posibilidades reales y excelentes de obtener una formación adecuada, con la finalidad de egresar profesionales integrales que contribuyan a hacer de nuestro país, una nación capaz de producir bienes y servicios adecuados a las necesidades de su población (pág. 87). 
La educación superior en Venezuela ha logrado configurarse como un subsector educativo con innegables contribuciones positivas al país, cumpliendo un papel decisivo tanto en la generación de conocimientos, la formación de profesionales e intelectuales al servicio de la comunidad; así como en el fortalecimiento de los valores propios a la consolidación de una sociedad democrática. Aún más, cuando en la actualidad enfrenta "un gran reto," pues debe convocar a la formación de un hombre más humano, más sensible a su entorno; en tal sentido, debe plantearse como meta principal la creación de valores éticos entre los cuales deben destacar la eficacia, la eficiencia, la transparencia y la equidad.

En esta sociedad cambiante, el conocimiento se convierte en uno de los recursos más importantes de desarrollo. En este sentido, la educación superior esta llamada a la formación del hombre integral: crítico, auto responsable, y sobre todo con una formación científica que este evolucionando constante y progresivamente como avance la información, momento de cambios quizás radicales, donde todavía hay muchos que sufren de parálisis paradigmática.

\section{Educación en Valores desde el Contexto Universitario.}

Hoy más que nunca nuestra sociedad reclama de sus instituciones de educación superior, una formación en valores de sus jóvenes, que opere como eje transversal dentro de los planes de estudio, ante estas exigencias se deben asumir de forma obligatoria y con sentido de responsabilidad, para los estudiantes universitarios, una educación que incluya este eje. Desde esta perspectiva, la universidad como rectora del saber y del conocimiento tiene como fin divulgar valores morales, mismos que no deben quedar solo enmarcados puramente en conceptos, sino que deben practicarse en la cotidianidad como acción inmediata, de la práctica pedagógica del docente.

Como punto de partida es necesario decir que toda universidad pública o privada tiene su filosofía para concebir la educación en valores y lograr con 
ello la formación integral de sus estudiantes, por ello en estos tiempos, es imprescindible ahondar la esencia de la filosofía de toda universidad, con la intención de delinear acertadamente la concepción humana desde los postulados de la complejidad, dado que a partir de los valores, principios e ideales que están inscritos en la misión y la visión del legado de toda universidad, se requiere que toda la educación esté basada en los valores, de manera que permita a los estudiantes tener una actitud reflexiva, de juicio y de una conciencia en su tarea, asimismo, tener muy claro su visión de su futuro y disponerse a enfrentar los retos que demanda la sociedad postmoderna.

Con relación a lo planteado, Berkowitz, (1998), afirmó que el papel fundamental de la educación no es solamente desarrollar en los estudiantes aprender a conocer, aprender a hacer, aprender a ser, sino que es relevante aprender a vivir juntos, construir la sociedad que necesita el país, desde la perspectiva de los valores.

Por lo tanto, para lograr este objetivo, no se puede seguir haciendo más de lo mismo, es necesario potenciar el desarrollo de principios y modos propios de actuar del ser humano, en concordancia con la normas que rigen la sociedad En atención a lo planteado, se requiere que la educación asuma que la formación de valores éticos y morales en los estudiantes universitarios; es una necesidad irrevocable, que cobra fuerza cuando la universidad cumple con su cometido cuando activa la conciencia del estudiante, propicia la construcción de su identidad, en relación a los valores asumidos a partir de sus creencias, le motiva a la disposición de compromiso, servicio y respeto por el mismo y por los demás, le hace sentir orgullo por su desempeño y apreciar lo que representa la adquisición del conocimiento.

Es por ello, que el estudiante universitario se edifica, a partir de la relación que crea con la universidad, como institución creadora y autora de cultura, dado que los estudiantes universitarios la visualizan no sólo como parte del proceso de formación académica, sino también como parte de la 
colectividad, identidad que se determina cuando el estudiante universitario establece una relación con su medio educativo, ya que su identidad del yo se alimenta de la identidad colectiva.

Ya lo mencionan algunos autores, que la identidad se inicia al retomar y recrear desde el contexto cultural y profesional, las acciones que realizan los sujetos y sus expectativas sociales y culturales, como son sus valores y afirmaciones, es decir, las representaciones y significados que se ubican de forma constante en la vida diaria, alcanzaran la conciencia sociocultural y se verá reflejada por medio de los valores morales que dispongan, considerando a la vez sus habilidades y estilos de vida de acuerdo a los cambios sociales y culturales. A tal efecto, Carrizales (2001), señala:

Ser universitario implica sentirse protagonista en la creación de un mejor mundo posible ¿si no es eso, ¿qué es?, ¿acaso basta estudiar, trabajar, cobrar en la Universidad para ser universitario? No, ello implica estar, pero no ser. Se puede estar sin ser. Ser Universitario implica sentirse, reconocerse, expresarse como universitario (pág. 61).

Por su parte, González (2009) complementa esta idea enfatizando la visión filosófica y humanista, como componente de la identidad en los universitarios:

La universidad no sólo debe cambiar para adaptarse sino también y sobre todo, para preparar un porvenir conforme a una cierta concepción filosófica y humanista de la vida en sociedad. La educación debe formar ciudadanos activos capaces de dominar el progreso tecnológico para darle sentido a la vida intelectual y colectiva, para respetar el equilibrio del planeta, hacer reinar la paz, reducir la violencia y forjar un verdadero proyecto de sociedad (pág. 94).

Con ello reconocemos que el sentido de la identidad es la conciencia que tiene todo estudiante de pertenecer a una universidad, así como el significado de valor que resulte de ello, esta identificación debe ser tomada 
con un mayor compromiso y utilizar los conocimientos académicos adquiridos, durante su formación universitaria, para darle solución a los problemas de una comunidad.

Sin embargo, tener identidad universitaria no equivale únicamente a pertenecer a una comunidad universitaria, sino que, es el resultado de todo un proceso social que involucra tener claro y sobre todo compartir los valores éticos y morales, como su historia, sus costumbres, sus símbolos, su misión y visión, el quehacer de la cotidianidad, así como la responsabilidad social que encierra el ser y quehacer de la universidad, por lo tanto tener identidad universitaria es ser parte de una institución actuando de acuerdo a lo expuesto en su visión y misión.

Por lo tanto, cuando se habla de valores, especialmente en el contexto educativo universitario, se hace de un modo relativamente intuitivo ya que la educación en valores en este nivel tiende a propiciar el desarrollo de ciertas actitudes en los estudiantes que faciliten la formación en ellos de un juicio moral autónomo que consolide una genuina educación en valores, modelada por el docente durante su práctica pedagógica.

Así mismo, es vital la concreción de la universidad como un escenario de producción social, ya no sea vista entonces dentro de la concepción tradicional de "recinto cerrado" o "claustro de conocimiento" sino como una organización o sistema abierto al flujo social y más aún, con capacidad para dar respuestas a las necesidades y demandas de un contexto epocal donde lo permanente es el cambio, la transformación y el desafío es permanecer a la vanguardia del conocimiento. Mínguez (1995) dicen:

"...la sociedad moderna delega en la educación superior la función de desarrollar en los estudiantes las competencias necesarias para actuar responsablemente en la sociedad lo cual implica, entre otros aspectos, el desarrollo de unos planes de estudio en el que los contenidos teóricos se complementen con el desarrollo de habilidades y competencias, como son: 
trabajo en equipo, capacidad de resolución de problemas, creatividad..." (pág. 52).

La realidad actual que se vive, urge individuos integrales y proactivos con competencias que les permitan actuar de manera eficaz en la sociedad. En este sentido, se vislumbra la importancia que tiene la formación integral de los estudiantes; a estos se les debe guiar y orientar sistemáticamente en todas las etapas que transita promoviendo e integrando la ética y los valores morales; elementos fundamentales que debe ser tomado en cuenta en los componentes del currículo universitario.

No es un secreto para nadie que los cambios que sean dados en sociedad han traído como consecuencia una profunda crisis de valores. Es por ello, que nuestras casas de estudios deben poner el acento supremo en aumentar su capacidad para servir en medio de la incertidumbre, para transformarse y provocar cambios en función de atender las necesidades sociales, innovando nuevas estrategias para generar el conocimiento como uno de los recursos más importantes de desarrollo basados en dimensiones de moralidad y espiritualidad. Solo de esta manera se garantiza que los estudiantes comprendan, conciencien y asuman los emergentes cambios que se dan en su estructura a nivel social, económico, político y cultural, así como las diversas formas de abordarlos con una actitud crítica y responsable.

En referencia a lo planteado Sullivan (2008) expresa:

"el profesor es uno de los componentes esenciales del proceso educativo y en quien descansa la máxima responsabilidad de desarrollo integral de los estudiantes... es el recurso humano más importante, quien hace posible la misión de las universidades, no se puede aspirar a formar profesionales capaces, si los docentes no son conscientes de su responsabilidad y están aptos para ello." (pág. 162).

Lo comentado, por el autor permite dilucidar que el docente universitario en los actuales momentos, debe ser una guía para el estudiante en el proceso 
de aprendizaje, no como un simple transmisor de conocimientos sino bajo una relación afectiva, dialógica y valorativa. En tal sentido, estamos hablando de que todo docente debe tener un perfil profesional que lo caracterice: conjunto de roles, de conocimientos, habilidades y destrezas, actitudes y valores necesarios que posea para el desempeño de su profesión conforme a las diversas condiciones del contexto donde interactúa.

Es pertinente que el docente realice de manera coherente la docencia, la investigación y la extensión; para lograr la activa participación de los participantes en los asuntos comunitarios y obtener un continuo saber y el aprender, de ese nuevo saber, le permita aprender a desaprender y aprender a aprender; dicho de otra forma, debe planificar los contenidos programáticos enmarcados en la realidad regional, local y por ende nacional. De esta manera, promueve la investigación, desde contextos y escenarios reales desarrollando en los estudiantes una actitud crítica ante las diversas necesidades y problemas presentes en nuestra geografía. En este sentido la praxis pedagógica del docente universitario no bebe estar descontextualizada ya que es la forma de contribuir a la transformación del país que queremos, promoviendo principios y valores que van en función del bien común.

Morín citado por Martí (2003) acota;

"El conocer la realidad se relaciona con lo ético, lo estético y también con la vida. Sin embargo, cuando se conoce parcialmente, sin ver lo que "está tejido en conjunto" se puede olvidar o ignorar las consecuencias del propio conocimiento, dejando de lado la responsabilidad y la solidaridad, valores fundamentales para interactuar como sujeto social". (pág. 57).

Desde esta postura se puede decir, que la ética y los valores morales son esenciales en el desarrollo educativo y cultural de una comunidad, sentada sobre bases democráticas para la formación de individuos solidarios, participativos, proactivos y respetuosos. 
En conclusión, se puede decir, que el docente debe tener un perfil integral para lograr los fines de la educación superior, no solo debe limitarse a transmitir conocimientos, para desarrollar habilidades intelectuales y prácticas, sino que junto a esto, tiene que contribuir a la formación de una persona digna, libre, respetuosa, amante de la vida, la naturaleza, la verdad, entre otros valores esenciales. Esto demuestra que el docente tiene una sólida formación axiológica que regula su actuación profesional y que lo caracteriza como un profesional con capacidad para comprender su papel protagónico en la sociedad, asumiéndolo con responsabilidad, honestidad y tolerancia.

Este Perfil responde de manera significativa a las transformaciones y desafíos presentes en la sociedad, ya que logra una nueva actitud tanto del docente como de los estudiantes en la forma de acceder y utilizar el conocimiento con responsabilidad ética, social y ambiental que le permita actuar de manera crítica y proactiva en el desarrollo de país. Asimismo, se logra el respecto por los derechos humanos, el respeto a las identidades, a la cultura, la paz y unidad. Se promueve la participación activa de estos dos actores (docente-estudiante) en las comunidades fortaleciendo el desarrollo endógeno.

\subsection{Valores a Resaltar en la Educación Superior}

La formación de valores constituye un problema pedagógico complejo solamente comprensible a partir de un análisis psicológico de la naturaleza del valor en su función reguladora de la actuación humana.

En su conceptualización psicológica el valor debe ser analizado teniendo en cuenta su naturaleza objetiva-subjetiva.

El valor en tanto significación de un hecho es al mismo tiempo objetivo y subjetivo ¿Quién atribuye significado? El ser humano. Por tanto, el valor como significado atribuido tiene una naturaleza subjetiva toda vez que existe individualmente en los seres humanos capaces de valorar; pero al mismo 
tiempo tiene una naturaleza objetiva que constituye parte de la realidad social e histórica en la que se desarrolla el ser humano.

En este sentido, los valores tienen además de una existencia individual, una existencia supraindividual toda vez que forman parte de la realidad social como relación de significados entre los hechos de la vida social y las necesidades de la sociedad, e histórica como sistema de valores oficialmente instituidos en una sociedad concreta.

La comprensión de la naturaleza objetiva-subjetiva del valor es fundamental para su educación. Porque tanto los valores más trascendentes para una sociedad como la igualdad, la justicia, la solidaridad, como los valores más específicos, por ejemplo, en el orden profesional, el amor a la profesión, la responsabilidad, son reflejados por cada persona de manera diferente en función de su historia individual, de sus intereses, capacidades; quiere decir que no siempre los valores jerarquizados oficialmente por una sociedad como los más importantes (existencia objetiva del valor) son asumidos de igual manera por los miembros de la sociedad (existencia subjetiva del valor). Esto ocurre porque la formación de valores en lo individual no es lineal y mecánica, sino que pasa por un complejo proceso de elaboración personal en virtud del cual los seres humanos, en interacción con el medio histórico-social en el que se desarrollan, construyen sus propios valores.

La diferencia entre la existencia objetiva y subjetiva del valor y su expresión en la conciencia del hombre es explicada por Leontiev (2014) a través de sus conceptos, significados y sentido personal.

En este sentido, González (2000) plantea que "Ningún contenido que no provoque emociones, que no estimule nuestra identidad, que no mueva fibras afectivas, puede considerarse un valor" (pág. 35), porque este se instaura a nivel psicológico de dos formas: los valores formales que regulan el comportamiento del hombre ante situaciones de presión o control externos, y creo que no son los que debemos formar, y los valores personalizados, 
expresión legítima y auténtica del sujeto que los asume, y que son, los valores que se deben fomentar en toda la sociedades.

De esta manera un estudiante universitario es responsable no porque conozca la importancia del valor responsabilidad o las circunstancias lo obliguen a ser responsable, sino porque siente la necesidad de actuar responsablemente. La responsabilidad en este caso, deviene un motivo de la actuación. Por tanto, sólo cuando los valores constituyen motivos de la actuación del sujeto se convierten en verdaderos reguladores de su conducta.

\section{Metodología}

La metodología consistió en el análisis documental de autores de amplia trayectoria en el ámbito del estudio. Para el primer caso, el análisis documental, bajo el diseño bibliográfico puro se realizó a través de la técnica de revisión de documentos dirigida a obtener información en el estudio de cualquier caso. El uso más importante de los documentos es corroborar y aumentar las evidencias encontradas en otras fuentes, por ello la información requerida se ubicó en una serie de documentos vinculados a la temática de la investigación, dando el soporte teórico del mismo, desarrollando las categorías valores, educación y universidad a fin de ampliar la información de manera objetiva y categórica dentro de la investigación.

Ahora bien, el tipo de estudio se desarrolló bajo el enfoque del paradigma interpretativo, con una metodología de carácter cualitativo. El uso de la metodología cualitativa en esta investigación se justifica porque el tema de los valores en educación posee un alto contenido subjetivo, así no se limitaría la información subjetiva que pudo ser obtenida de sujetos informantes. En cuanto al método de investigación, éste se basa en el estudio de casos.

En lo referido a las técnicas de recolección de la información, en el método de investigación de estudios de casos, Yin (1994) recomienda la utilización de múltiples fuentes de información y el cumplimiento del principio 
de triangulación para garantizar la fiabilidad interna de la investigación. Esto permite verificar si la información obtenida a través de las diferentes fuentes de información guarda relación entre sí (principio de triangulación); es decir, si desde diferentes perspectivas convergen los efectos explorados en el fenómeno objeto de estudio.

Para facilitar la fiabilidad, la metodología cualitativa cuenta con dos técnicas: la triangulación (realizada con distintas fuentes de información, diferentes perspectivas teóricas, con distintos observadores, de diferentes procedimientos metodológicos, entre otros) y las grabaciones de audio y video (Martínez, 2006). Para efectos de esta investigación, se utilizaron ambas técnicas.

\subsection{Análisis de los Resultados}

Se puede decir, que el docente debe tener un perfil integral para lograr los fines de la educación superior, no solo debe limitarse a transmitir conocimientos, para desarrollar habilidades intelectuales y prácticas, sino que junto a esto, tiene que contribuir a la formación de una persona digna, libre, respetuosa, amante de la vida, la naturaleza, la verdad, entre otros valores esenciales. Esto demuestra que el docente tiene una sólida formación axiológica que regula su actuación profesional y que lo caracteriza como un profesional con capacidad para comprender su papel protagónico en la sociedad, asumiéndolo con responsabilidad, honestidad y tolerancia. que responda de manera significativa a las transformaciones y desafíos presentes en la sociedad, que pueda lograr una nueva actitud tanto del docente como de los estudiantes en la forma de acceder y utilizar el conocimiento con responsabilidad ética y social que le permita actuar de manera crítica y proactiva en el desarrollo de sus potencialidades.

Asimismo, se logra el respecto por los derechos humanos, el respeto a las identidades, a la cultura, la paz y unidad. Se promueve la participación 
activa de estos dos actores (docente-estudiante) en las comunidades fortaleciendo el desarrollo endógeno.

En definitiva, el docente promueve en los estudiantes, la autonomía, la responsabilidad; desde la participación activa de éste en su proceso de aprendizaje, construyendo conocimiento en forma individual y social. Esta nueva postura permite que el participante sea reflexivo, crítico involucrados con la realidad nacional.

\section{Conclusiones Finales}

Vivimos en un mundo cambiante donde los referentes éticos y morales, es decir, los valores socialmente dominantes tienden a variar y a modificarse creando, en muchas ocasiones, contradicciones de difícil resolución.

Es por ello que, la educación superior, como reflejo de la sociedad de la que forma parte, no es ajena a estos cambios y así, dentro de este contexto, en los últimos años uno de los paradigmas de debate en torno a la educación ha estado centrado en el ámbito axiológico.

Sin embargo, los valores, o más bien la educación en valores, se ha erigido como un núcleo de análisis y discusión desde un universo heterogéneo de planteamientos, donde los mismos hacen referencia a modelos ideales de actuar y de existir que el ser humano aprecia, desea y busca, a través de los cuales interpreta el mundo y da significado a su existencia.

Debido a su consideración de ideal, de algo a lo que se tiende, poseen una naturaleza abstracta e intangible y sólo se hacen explícitos a través de las conductas o los modos de comportarse que manifiesta una persona ante determinadas situaciones. En este sentido, la tendencia (o predisposición aprendida) a comportarse de una manera ante determinadas realidades vividas: problemas, ideas, situaciones, personas o acontecimientos, recibe el nombre de actitud. 
Es relevante determinar cómo surgen los valores, no se produce a través del intelecto, por el contrario, responden a la lógica del sentimiento y no únicamente desde un ideal de justicia sino también desde ideales de felicidad. Desde este punto de vista, el papel y la actitud del docente como profesional, constituye el agente más relevante en la transmisión de valores en un aula de clase. Aspectos como la relación que establece con los alumnos, el clima de clase que propicia, la metodología utilizada, entre otros, cobran especial relevancia.

\section{Referencias}

Berkowitz, M. (1998). Educar la persona moral en su totalidad. En Educación, Valores y Democracia, OEI, Edit. FotoJAE, S.A., Madrid.

Carrizales, C. (2001). Paisajes Universitarios. Cuernavaca, Morelos: UAEM. González, B. (2009). Significados axiológicos en la actuación de los estudiantes del programa de preescolar de la UPEL-IPB. Tesis Doctoral. UFT. Cabudare, Venezuela.

González, V. (2000). La Educación en Valores en el Currículum Universitario. Un Enfoque Psicopedagógico para su estudio. Educación Médica Superior, Revista Cubana Educación Media Superior, vol.14, n.1, pp. 74-82, ISSN: 1561-2902. Recuperado de: http://scielo.sld.cu/scielo.php?script=sci arttext\&pid=S0864$\underline{21412000000100010}$

Leontiev, C. (2014). Valores de Cada Día. Venezuela: Ediciones San Pablo Martí J. (2003). La Ciencia y la Técnica en el Desarrollo de Valores desde el Pensamiento económico.

Martínez, M. (2004). Como hacer un buen proyecto de tesis con metodología cualitativa. [Documento en línea]. Recuperado de:

http://prof.usb.ve/miguelm/proyectotesis.html 
Romero, C. (2004). Valores humanos y educación en/para la tolerancia. Revista AULA, No. 7, Ediciones de la Universidad de Salamanca.

Sullivan P. (2008). La complejidad del Conocimiento y el Problema de la Educación en el Siglo XXI.

Yin, R. (1994). Case study research: design and method. Reino Unido. Sage Publications. 


\section{María Lastenia Palacios Palacio}

e- mail: marialasteniapalacios@hotmail.com

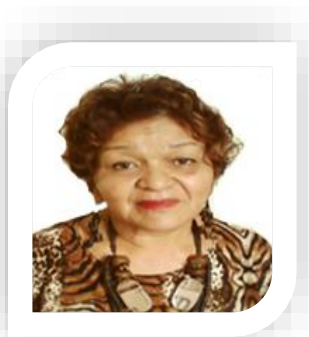

Nací en Barquisimeto, estado Lara, Venezuela. Actualmente estudiante del Postdoctorado en estudios Libre en la Universidad Fermín Toro con título de Pregrado en ABOGADA, egresada de la Universidad Fermín Toro. Con Doctorado en Ciencias de la Educación, de la Universidad Fermín Toro, Postgrado en Ciencias Penales y Criminológicas en la Universidad Yacambú. Postgrado en Procesal Penal en la Universidad Fermín Toro. Postgrado en Procesal Civil en la Universidad Fermín Toro. Diplomado en Medios Alternativos de Resolución de Conflictos en el Colegio de Abogados, del Estado Lara. Actualmente terminé Académicamente un Doctorado en Derecho Constitucional y una Maestría en Derecho Constitucional y Derechos Fundamentales en la Universidad Caribbean International de Curazao, ambos por On-Line.

El contenido de este manuscrito se difunde bajo una Licencia de Creative Commons ReconocimientoNoComercial-Compartirlgual 4.0 Internacional 